(2) Open Access Full Text Article

\title{
Synergistic effects of antimicrobial peptide DP7 combined with antibiotics against multidrug- resistant bacteria
}

This article was published in the following Dove Press journal:

Drug Design, Development and Therapy

22 March 2017

Number of times this article has been viewed

\section{Xiaozhe $\mathrm{Wu}^{\prime}$ \\ Zhan $\mathrm{Li}^{\prime}$ \\ Xiaolu $\mathrm{Li}^{2,3}$ \\ Yaomei Tian' \\ Yingzi Fan' \\ Chaoheng $\mathrm{Yu}^{\prime}$ \\ Bailing Zhou' \\ Yi Liu ${ }^{4}$ \\ Rong Xiang ${ }^{5}$ \\ Li Yang'}

'State Key Laboratory of Biotherapy/ Collaborative Innovation Center of Biotherapy, West China Hospital,

Sichuan University, ${ }^{2}$ International Center for Translational Chinese Medicine, Sichuan Academy of Chinese Medicine Sciences, Chengdu, ${ }^{3}$ Department of Plastic and Burn Surgery, Affiliated Hospital of Southwest Medical University, Luzhou, ${ }^{4}$ Department of Microbial Examination, Sichuan Center for Disease Control and Prevention, Chengdu, ${ }^{5}$ Nankai University School of Medicine, Tianjin, People's Republic of China

Correspondence: Li Yang State Key Laboratory of Biotherapy/ Collaborative Innovation Center of Biotherapy, West China Hospital, Sichuan University, 18 North Renming Road, Chengdu 610014, People's Republic of China

Email yl.tracy73@gmail.com

\begin{abstract}
Antibiotic-resistant bacteria present a great threat to public health. In this study, the synergistic effects of antimicrobial peptides (AMPs) and antibiotics on several multidrugresistant bacterial strains were studied, and their synergistic effects on azithromycin (AZT)resistance genes were analyzed to determine the relationships between antimicrobial resistance and these synergistic effects. A checkerboard method was used to evaluate the synergistic effects of AMPs (DP7 and CLS001) and several antibiotics (gentamicin, vancomycin [VAN], AZT, and amoxicillin) on clinical bacterial strains (Staphylococcus aureus, Pseudomonas aeruginosa, Acinetobacter baumannii, and Escherichia coli). The AZT-resistance genes (ermA, ermB, ermC, $m e f A$, and $m s r A$ ) were identified in the resistant strains using quantitative polymerase chain reaction. For all the clinical isolates tested that were resistant to different antibiotics, DP7 had high antimicrobial activity ( $\leq 32 \mathrm{mg} / \mathrm{L})$. When DP7 was combined with VAN or AZT, the effect was most frequently synergistic. When we studied the resistance genes of the AZT-resistant isolates, the synergistic effect of DP7-AZT occurred most frequently in highly resistant strains or strains carrying more than two AZT-resistance genes. A transmission electron microscopic analysis of the $S$. aureus strain synergistically affected by DP7-AZT showed no noteworthy morphological changes, suggesting that a molecular-level mechanism plays an important role in the synergistic action of DP7-AZT. AMP DP7 plus the antibiotic AZT or VAN is more effective, especially against highly antibiotic-resistant strains.
\end{abstract}

Keywords: antimicrobial peptide, drug synergy, resistant bacteria

\section{Introduction}

With the ongoing use of antibiotics, increasing numbers of bacteria are becoming resistant to them. Strategies to address this problem include the identification of new antibiotics, the application of the principles of microecology, or the development of antimicrobial peptides (AMPs). AMPs are one of the most promising antibacterial drugs that can be substituted for antibiotics because they have advantages such as high antimicrobial efficacy ${ }^{1-3}$ and broad-spectrum activity. ${ }^{1,4}$ Another way to resolve the problem of multidrug-resistant bacteria is by combining different drugs. The effects of AMPs combined with antibiotics often exceed those of the individual drugs, whether in antibacterial activities ${ }^{5-8}$ or in interruption of biofilm formation. ${ }^{9-13}$ Drug design research faces a long and difficult challenge to overcome drug resistance, whereas drug combinations require no drug modification and lower doses can be used while enhancing the therapeutic potential. ${ }^{14,15}$

DP7 is a recently designed AMP derived from Bac2A and has demonstrated good broad-spectrum antimicrobial activity and safety in previous studies. ${ }^{16}$ CLS001 is 
an AMP that is currently under investigation in a Phase III clinical trial. ${ }^{17,18}$ In this study, we evaluated the in vitro antimicrobial activities of gentamicin (GEN), amoxicillin (AMO), azithromycin (AZT), and vancomycin (VAN), alone and in combination with DP7 or CLS001, against antibioticresistant Staphylococcus aureus, Pseudomonas aeruginosa, Acinetobacter baumannii, and Escherichia coli strains. The AZT-resistance mechanism was also examined to clarify the synergistic mechanism involved and the traits of multidrugresistant bacteria in general.

\section{Materials and methods}

\section{Bacterial strains}

The clinical isolates used in this study were isolated and obtained from the Southwest Hospital of China and the Sichuan Center for Disease Control and Prevention; duplicate sample from one patient was excluded. The species were confirmed using the Bacteria Identification Microbial DNA qPCR Multi-Assay Kit (Qiagen NV, Venlo, the Netherlands).

\section{Antimicrobial agents}

The peptides were synthesized with Fmoc chemistry by Shanghai Applied Protein Technology Co., Ltd (Shanghai, People's Republic of China). VAN hydrochloride was purchased from Gentle Pharmaceutical Corporation Kashin Medicines Co., Ltd (Taiwan, Republic of China), AZT powder was purchased from Chen Mei Chemical Co., Ltd (Zhengzhou, People's Republic of China), gentamicin sulfate was purchased from Hubei Pharmaceutical Co., Ltd (Hubei, People's Republic of China), and AMO was purchased from Kunming Baker Norton Pharmaceutical Co., Ltd (Kunming, People's Republic of China).

\section{Susceptibility test}

The minimum inhibitory concentrations (MICs) of the antimicrobial drugs were determined using the broth microdilution method, according to Clinical and Laboratory Standards Institute (CLSI) file M7-A7. Serial dilutions of the peptides were prepared in Müller-Hinton broth, and $100 \mu \mathrm{L}$ of each concentration was added to triplicate wells in a 96-well flat-bottom tissue culture plate. The bacteria were grown on Müller-Hinton agar for 24 hours. A few colonies were diluted to $\sim 1 \times 10^{6} \mathrm{cfu} / \mathrm{mL}$ with MüllerHinton broth, and $100 \mu \mathrm{L}$ was added in triplicate to each well containing the antimicrobial drugs. The final drug concentrations ranged from $256 \mathrm{mg} / \mathrm{L}$ to $0.25 \mathrm{mg} / \mathrm{L}$. The suspensions were then incubated at $37^{\circ} \mathrm{C}$ for 20 hours.
Bacterial concentration was calculated through optical density, and the lowest concentration causing $80 \%$ growth inhibition relative to the growth of the control was deemed to be the MIC.

\section{Checkerboard assay}

The activities of the AMPs in combination with the antibiotics were analyzed using the checkerboard broth dilution method $^{19}$ to determine the fractional inhibitory concentration indices (FICIs), calculated as: FICI $=$ MIC of drug A in combination/MIC of drug A alone + MIC of drug B in combination/MIC of drug $\mathrm{B}$ alone. Twice the maximum concentration was used in calculation if the MIC was out of measurement in this study. The calculated FICI was interpreted as synergistic (FICI $\leq 0.5)$, additive $(0.5<$ FICI $<1)$, indifferent $(1 \leq \mathrm{FICI}<4.0)$, or antagonistic $(\mathrm{FICI} \geq 4.0) .{ }^{20}$

\section{Gene identification using quantitative polymerase chain reaction}

Total DNA was collected from the bacteria using the DNeasy Blood \& Tissue Kit (Qiagen NV). DNA concentration was determined with a Thermo Scientific NanoDrop 2000 spectrophotometer. DNA samples with concentrations (measured at $\left.A_{260}\right)>10 \mathrm{ng} / \mathrm{mL}$ and $A_{260} / A_{280}$ ratios $>1.8$ were used in the quantitative polymerase chain reaction (qPCR) assay. Bacterial genes, VAN-resistance genes, and AZT-resistance genes were identified with the Microbial DNA qPCR MultiAssay Kits from Qiagen NV. The level of gene expression was determined as the difference between the quantification cycle $(\mathrm{Cq})$ value of the target sample and that of the nontemplate control (NTC), $\mathrm{Cq}>\mathrm{NTC}-3$ was deemed negative for gene expression. $\mathrm{Cq}<\mathrm{NTC}-6$ was deemed positive for gene expression; and $\mathrm{NTC}-6>\mathrm{Cq}>\mathrm{NTC}-3$ was deemed inconclusive.

\section{Transmission electron microscopy}

Mid-logarithmic phase $S$. aureus cells $\left(1 \times 10^{8}\right)$ were treated with DP7, AZT, or DP7-AZT, and a no-peptide control was included. After incubation for 60 minutes, the bacteria were pelleted by centrifugation at $900 \times g$ for 10 minutes and washed twice with $1 \mathrm{M}$ phosphate-buffered saline. The cells were then fixed with $2.5 \%$ glutaraldehyde/ phosphate-buffered saline at $4{ }^{\circ} \mathrm{C}$ overnight. The prepared bacterial samples were sent to the Center of Forecasting and Analysis of Sichuan University (Sichuan, People's Republic of China) for imaging with transmission electron microscopy (TEM, Tecnai G2 F20 S-TWIN, FEI). 


\section{Results \\ MIC distribution}

The MIC of each antibiotic and AMP was determined using the broth microdilution method before the synergy test, and the highest concentration obtained was $256 \mathrm{mg} / \mathrm{L}$. Clinical isolates of $S$. aureus (SAU series), P. aeruginosa (PAER series), A. baumannii (ABA series), and E. coli (ECO series) were examined (Table 1). Most bacterial strains were resistant to the tested antibiotics (GEN, VAN, AMO, and AZT), and GEN and AMO showed negligible activity against these strains. Only strain SAU4 was sensitive to VAN and AZT. Both AMPs CLS001 and DP7 showed strong activity against the clinically isolated strains in this study, while the antimicrobial activity of DP7 was slightly better than that of CLS001.

\section{Synergy assay}

The microdilution checkboard method was used to evaluate the synergistic effects of the antibiotics combined with the AMPs, which were determined as FICIs (Table 2). The effects of the DP7-VAN combination were synergistic against $50 \%$ of all the isolates tested and additive against $40 \%$ of the isolates. The effects of the DP7-AZT combination were synergistic against $60 \%$ of the isolates and additive against $20 \%$ of the isolates. These two combinations were the most promising of those tested against the antibiotic-resistant bacteria in this study. The percentages of isolates affected synergistically and additively by DP7-AMO were $20 \%$ and $10 \%$, respectively, whereas the DP7-GEN combination synergistically affected only $20 \%$ of isolates and additively

Table I MICs of clinically isolated S. aureus, P. aeruginosa, A. baumannii, and $E$. coli strains

\begin{tabular}{lllllll}
\hline Isolates & \multicolumn{2}{l}{ MIC $(\mathbf{m g} / \mathbf{L})^{\mathbf{a}}$} & & & & \\
\cline { 2 - 7 } & DP7 & CLS00 I & VAN & GEN & AZT & AMO \\
\hline ABAI & 8 & $4-16$ & $16-32$ & $>256$ & $32-128$ & $>256$ \\
ABA2 & $8-16$ & $8-32$ & $16-32$ & $>256$ & $>256$ & $>256$ \\
ABA3 & $4-8$ & $8-16$ & $16-32$ & $>256$ & $64-256$ & $>256$ \\
SAU2 & 32 & 64 & 0.5 & $>256$ & $>256$ & $>256$ \\
SAU7 & 32 & 128 & 0.25 & $>256$ & 128 & $>256$ \\
SAU8 & 32 & 64 & 0.5 & $>256$ & 128 & $>256$ \\
ECOI & $4-8$ & 16 & 32 & $>256$ & 64 & $>256$ \\
ECO2 & $4-8$ & $8-16$ & $32-64$ & $>256$ & $>256$ & $>256$ \\
ECO3 & 8 & $16-32$ & $64-128$ & $>256$ & $>256$ & $>256$ \\
PAERI & $4-8$ & $4-16$ & 32 & $>256$ & $32-128$ & $>256$ \\
PAERI0 & 4 & $2-16$ & 32 & $>256$ & $32-128$ & $>256$ \\
PAERII & 8 & $2-16$ & $16-32$ & $>256$ & $64-128$ & $>256$ \\
\hline
\end{tabular}

Note: ${ }^{\mathrm{M}} \mathrm{MIC}$ ranges in triplicate tests.

Abbreviations: MIC, minimum inhibitory concentration; S. aureus, Staphylococcus aureus; P. aeruginosa, Pseudomonas aeruginosa; A. baumannii, Acinetobacter baumannii; E. coli, Escherichia coli; VAN, vancomycin; GEN, gentamicin; AZT, azithromycin; AMO, amoxicillin. affected no isolates. The synergistic activities of CLS001 combined with these antibiotics were also tested and compared with those of DP7. The synergistic effect rates were $20 \%$ for CLS001-VAN, $20 \%$ for CLS001-GEN, $10 \%$ for CLS001-AZT, and 40\% for CLS001-AMO. The effects of CLS001-VAN and CLS001-AZT were additive in 30\% and $50 \%$ of isolates, respectively, whereas no additive effect was observed for CLS001-GEN or CLS001-AMO. These results indicate that DP7 had a higher synergy rate than CLS001 when combined with VAN or AZT. Another remarkable result was that the combination of the two AMPs, DP7 plus CLS001, showed no synergistic effect on any bacterial isolate tested but had additive effects on $40 \%$ of isolates.

\section{Expanded synergy assay}

Because DP7-VAN and DP7-AZT had relatively high synergy rates, these two combinations were tested on ten more $S$. aureus and $P$. aeruginosa clinical strains to study the synergistic effects on gram-positive and gram-negative bacteria (Table 3). In $S$. aureus, DP7-VAN had synergistic effects on $40 \%$ of isolates and additive effects on another $40 \%$ of isolates. DP7-AZT had synergistic effects on $50 \%$ of isolates, but an additive on no isolate. In P. aeruginosa, DP7-VAN also showed high synergy rates, exerting a synergistic effect on $50 \%$ of isolates and an additive effect on $20 \%$ of isolates. However, DP7-AZT had a synergistic effect on only $10 \%$ of isolates and an additive effect on $30 \%$ of isolates. Although DP7-AZT showed low activity against $P$. aeruginosa, it showed relatively high activity against AZT-resistant $S$. aureus strains. DP7-VAN exerted synergistic effects against both $S$. aureus and $P$. aeruginosa strains that were either resistant to VAN or not.

\section{Resistance mechanism}

Isolates resistant to VAN or AZT were selected to analyze their resistance genes. The VAN-resistance genes vanB and vanC were analyzed with qPCR, but none of the VAN-resistant isolates tested was positive for either gene (data not shown). The occurrence of five AZT-resistance genes (ermA, ermB, ermC, $m e f A$, and $m s r A$ ) in the AZT-resistant isolates was investigated. Of the 13 AZT-resistant $P$. aeruginosa isolates, eleven carried ermB and six carried erm $C$ (all of which also carried ermB). Among the eleven $S$. aureus isolates, nine were resistant to AZT, and of these, four carried erm $A$, six carried ermB, six carried erm $C$, two carried both erm $A$ and erm $C$, three carried both ermB and erm $C$, and one carried erm $A$, ermB, and ermC. No erm $A$ gene was found in any $P$. aeruginosa isolate, and neither the mefA nor $m s r A$ gene was detected in any $P$. aeruginosa or $S$. aureus isolate in this study (Tables 4 and 5). 
Table $2 \mathrm{FICls}$ of antibiotics combined with DP7 or CLSOOI to treat clinically isolated S. aureus, P. aeruginosa, A. baumannii, and E. coli strains

\begin{tabular}{|c|c|c|c|c|c|c|c|c|c|}
\hline \multirow[t]{3}{*}{ Isolates } & \multicolumn{9}{|l|}{$\mathrm{FICl}^{\mathrm{a}}$} \\
\hline & \multicolumn{5}{|l|}{ DP7+ } & \multicolumn{4}{|c|}{ CLSOOI+ } \\
\hline & CLSOOI & VAN & GEN & AZT & AMO & VAN & GEN & AZT & AMO \\
\hline ABAI & 1.50 & 0.38 & 0.50 & 0.63 & 0.25 & 0.56 & 2.00 & 0.75 & 0.50 \\
\hline ABA2 & 0.75 & 0.75 & 2.00 & 1.00 & 0.50 & 0.63 & 1.00 & 0.75 & 0.50 \\
\hline ABA3 & 0.75 & 0.75 & 2.00 & 0.31 & 1.00 & 1.03 & 0.50 & 0.50 & 1.00 \\
\hline SAU2 & 1.00 & 0.52 & 1.00 & 0.01 & 1.00 & 0.25 & 1.00 & 0.19 & 0.50 \\
\hline SAU7 & 0.75 & 0.52 & 1.00 & 0.03 & 0.50 & 0.50 & 1.00 & 0.19 & 1.00 \\
\hline SAU8 & 1.00 & 0.52 & 0.50 & 0.38 & 1.00 & 0.375 & 1.00 & 0.19 & 1.00 \\
\hline ECOI & 1.25 & 0.56 & 2.00 & 0.75 & 1.00 & 1.50 & 1.00 & 1.00 & 1.00 \\
\hline $\mathrm{ECO} 2$ & 1.25 & 0.56 & 1.00 & 0.50 & 1.00 & 2.00 & 1.00 & 1.00 & 1.00 \\
\hline $\mathrm{ECO} 3$ & 1.50 & 0.50 & 1.00 & 1.00 & 1.00 & 1.50 & 0.50 & 1.00 & 1.00 \\
\hline PAERI & 0.63 & 1.00 & 4.00 & 0.04 & 1.00 & 0.38 & 1.00 & 0.56 & 1.00 \\
\hline PAERIO & 1.00 & 0.38 & 2.00 & 0.25 & 2.00 & 0.28 & 1.00 & 0.75 & 1.00 \\
\hline PAERII & 0.75 & 0.25 & 0.50 & 0.31 & 1.00 & 1.25 & 2.00 & 1.00 & 0.50 \\
\hline Synergistic, \% & 0 & 50 & 20 & 60 & 20 & 20 & 20 & 10 & 40 \\
\hline Additive, \% & 40 & 40 & 0 & 20 & 10 & 30 & 0 & 50 & 0 \\
\hline
\end{tabular}

Note: ${ }^{\mathrm{a}} \mathrm{FICl}$ is interpreted as synergistic $(\mathrm{FICl} \leq 0.5)$, additive $(0.5<\mathrm{FICl}<\mathrm{I})$, indifferent $(\mathrm{I} \leq \mathrm{FICl}<4.0)$, or antagonistic $(\mathrm{FICl} \geq 4.0)$.

Abbreviations: $\mathrm{FICl}$, fractional inhibitory concentration index; S. aureus, Staphylococcus aureus; P. aeruginosa, Pseudomonas aeruginosa; A. baumannii, Acinetobacter baumannii; E. coli, Escherichia coli; VAN, vancomycin; GEN, gentamicin; AZT, azithromycin; AMO, amoxicillin.

\section{Relationships between resistance genes, resistance, and synergy}

The relationships between the AZT-resistance mechanism, the MICs for AZT, and the FICIs for DP7-AZT were analyzed. As expected, isolates carrying more

Table 3 Susceptibility and synergy test results for DP7-VAN and DP7-AZT against clinically isolated strains of S. aureus and $P$. aeruginosa

\begin{tabular}{|c|c|c|c|c|c|}
\hline \multirow[t]{2}{*}{ Isolates } & \multicolumn{3}{|c|}{ MIC (mg/L) } & \multicolumn{2}{|l|}{$\mathbf{F I C I}$} \\
\hline & DP7 & VAN & AZT & DP7-VAN & DP7-AZT \\
\hline \multicolumn{6}{|c|}{ S. aureus drug-resistant strains } \\
\hline S5375 & $16-32$ & 64 & 32 & 0.38 & 0.19 \\
\hline SAU5 & 32 & 0.5 & $>256$ & 1 & I \\
\hline SAU9 & 16 & 0.5 & $>256$ & 0.28 & I \\
\hline S3487 & 32 & 8 & 64 & 1 & 1.06 \\
\hline S3750 & 32 & 8 & 4 & 0.5 & 1.5 \\
\hline S3396 & $4-32$ & 0.25 & 16 & 0.38 & 2 \\
\hline \multicolumn{6}{|c|}{ P. aeruginosa drug-resistant strains } \\
\hline PAER7 & 32 & $>256$ & 16 & 0.14 & 0.08 \\
\hline PAER6 & $>32$ & $>256$ & $>256$ & 0.28 & 0.53 \\
\hline PAER9 & 16 & 256 & 256 & 1.06 & 0.53 \\
\hline PAER2 & $16-32$ & $>256$ & 256 & 0.5 & 0.63 \\
\hline PAER4 & 16 & $>256$ & 256 & 0.56 & I \\
\hline PERA8 & 16 & $>256$ & 128 & 0.31 & I \\
\hline PAER5 & 16 & 64 & 64 & 0.53 & 1.03 \\
\hline P5I 28 & 16 & $>256$ & 32 & 1.02 & 1.5 \\
\hline PAER3 & $16-32$ & $>256$ & $>256$ & 0.5 & 2 \\
\hline P4477 & 16 & 128 & 4 & I & 2.06 \\
\hline
\end{tabular}

Abbreviations: VAN, vancomycin; AZT, azithromycin; S. aureus, Staphylococcus aureus; $P$. aeruginosa, Pseudomonas aeruginosa; MIC, minimum inhibitory concentration; $\mathrm{FICl}$, fractional inhibitory concentration index. erm-resistance genes had higher MICs for AZT. Isolate SAU2 carrying the ermA, ermB, and erm $C$ genes had the highest MIC for AZT $(>256 \mathrm{mg} / \mathrm{L})$. Of the isolates with two erm genes, $70 \%$ (7/10) were highly resistant to AZT (MIC $\geq 128 \mathrm{mg} / \mathrm{L}$ ). Of the isolates with one erm gene, $71.4 \%(5 / 7)$ had MICs $\geq 128 \mathrm{mg} / \mathrm{L}$. Isolates with no erm genes had MICs $\leq 32 \mathrm{mg} / \mathrm{L}$. The number of resistance genes also had some relationship with the FICI, in that isolates with more resistance genes showed lower FICIs. Of the nine isolates upon which DP7-AZT exerted a synergistic effect, seven $(77.8 \%)$ carried more than two resistance genes. Of the four isolates upon which DP7-AZT exerted an additive effect, two $(50 \%)$ carried two resistance genes. Among another nine isolates upon which DP7-AZT exerted an indifferent affect, only three (33.3\%) carried two resistance genes. Therefore, bacterial resistance to AZT increased as the number of AZT-resistance genes increased, and the likelihood of a synergistic DP7-AZT effect increased in parallel. The reason why DP7-AZT exerts a greater synergistic effect on more strongly AZT-resistant bacteria warrants further study.

Table 4 Resistance rates and synergy rates for each type of bacterium

\begin{tabular}{lllllll}
\hline Bacteria & ermA & ermB & ermC & mefA & msrA & Synergistic \\
\hline P. aeruginosa & $0 / 13$ & $11 / 13$ & $6 / 13$ & $0 / 13$ & $0 / 13$ & $6 / 13$ \\
S. aureus & $4 / 9$ & $6 / 9$ & $6 / 9$ & $0 / 9$ & $0 / 9$ & $4 / 9$ \\
\hline
\end{tabular}

Abbreviations: P. aeruginosa, Pseudomonas aeruginosa; S. aureus, Staphylococcus aureus. 
Table 5 Distribution of resistance genes in AZT-resistant isolates in ascending order of $\mathrm{FICl}$

\begin{tabular}{|c|c|c|c|c|c|c|c|}
\hline \multirow[t]{2}{*}{ Samples } & \multicolumn{5}{|c|}{ qPCR resistance genes expression ${ }^{a}$} & \multirow[t]{2}{*}{ AZT MIC (mg/L) } & \multirow{2}{*}{$\begin{array}{l}\text { Antimicrobial activity } \\
\text { DP7-AZT FICI }\end{array}$} \\
\hline & ermA & ermB & ermC & mefA & msrA & & \\
\hline Cq of NTC & 39.29 & 34.96 & 38.99 & 38.03 & 38.04 & & \\
\hline Positive control & + & + & + & + & + & & \\
\hline \multicolumn{8}{|l|}{ P. aeruginosa } \\
\hline PAERI & - & + & + & - & - & 128 & 0.04 \\
\hline PAER7 & - & + & + & - & - & 16 & 0.08 \\
\hline PAERIO & - & + & - & - & - & 128 & 0.25 \\
\hline PAERII & - & + & + & - & - & 128 & 0.31 \\
\hline PAER8 & - & + & + & - & - & 128 & 0.31 \\
\hline PAER6 & - & + & - & - & - & $>256$ & 0.53 \\
\hline PAER9 & - & + & - & - & - & 256 & 0.53 \\
\hline PAER2 & - & + & + & - & - & 256 & 0.63 \\
\hline PAER4 & - & + & - & - & - & 256 & 1.00 \\
\hline PAER5 & - & + & - & - & - & 64 & 1.03 \\
\hline P5I 28 & - & - & - & - & - & 32 & 1.50 \\
\hline PAER3 & - & + & + & - & - & $>256$ & 2.00 \\
\hline P4477 & - & - & - & - & - & 4 & 2.06 \\
\hline \multicolumn{8}{|l|}{ S. aureus } \\
\hline SAU2 & + & + & + & - & - & $>256$ & 0.01 \\
\hline SAU7 & + & - & + & - & - & 128 & 0.03 \\
\hline S5375 & - & - & + & - & - & 32 & 0.19 \\
\hline SAU8 & - & - & - & - & - & 128 & 0.38 \\
\hline S5768 & - & - & + & - & - & 64 & 0.63 \\
\hline SAU5 & - & - & + & - & - & $>256$ & 1.00 \\
\hline S3487 & - & + & - & - & - & 64 & 1.06 \\
\hline S3750 & - & + & - & - & - & 4 & 1.50 \\
\hline S3396 & - & + & + & - & - & 16 & 2.00 \\
\hline
\end{tabular}

Notes: aLevel of gene expression was determined by the difference between the Cq value of the target gene and that of the nontemplate control (NTC). - , $q \geq$ NTC $-6 ;+$, NTC $-6>$ Cq.

Abbreviations: AZT, azithromycin; $\mathrm{FICl}$, fractional inhibitory concentration index; qPCR, quantitative polymerase chain reaction; MIC, minimum inhibitory concentration; P. aeruginosa, Pseudomonas aeruginosa; S. aureus, Staphylococcus aureus.

\section{Morphological study of S. aureus strain S5375 treated with DP7-AZT}

The antimicrobial mechanism of DP7 acts by disrupting the bacterial cell wall and influencing the cell membrane. ${ }^{16}$ Therefore, the synergistic action of DP7-AZT on the bacterial morphology was investigated. S. aureus strain S5375, which is resistant to AZT but showed a synergistic response to DP7-AZT, was treated with $4 \times$ MICs of AZT for 60 minutes, and any damaging effects were observed with TEM (Figure 1). Compared with the bacteria in the control group, the bacteria treated with AZT had darker nuclei and clearer cell walls, which may be attributable to interrupted protein synthesis, with a consequent increased transcription activity in the nucleus and reduction in cell wall proteins. However, in the DP7-treated group, no nuclei were observed, the whole cytoplasm was a mosaic, and the cell edges were fuzzy. Interestingly, the cell walls of dividing cells were detached, indicating that cell wall synthesis was interrupted. However, the shape of the bacteria treated with DP7-AZT did not differ greatly from those treated with DP7 alone, which suggests that DP7 functions earlier than AZT. The only difference between the DP7-AZT-treated and DP7treated bacteria was that even some nondividing bacteria had no cell walls after treatment with DP7-AZT. This may be attributable to the combined action of the reduced cell wall proteins caused by AZT and the cell wall disruption caused by DP7. These results suggest that the synergistic mechanism of DP7-AZT acts at the molecular level.

\section{Discussion}

Many AMPs have been developed in recent years as alternatives to antibiotics to resolve the problem of antimicrobial resistance. ${ }^{21-23}$ The activity of one of these AMPs, DP7, against antibiotic-resistant clinical isolates was investigated in this study. The broad-spectrum, stable antimicrobial activities of DP7 and CLS001 were confirmed in susceptibility tests against $S$. aureus, $P$. aeruginosa, A. baumannii, and E. coli isolates. Although the MICs of the AMPs are usually 

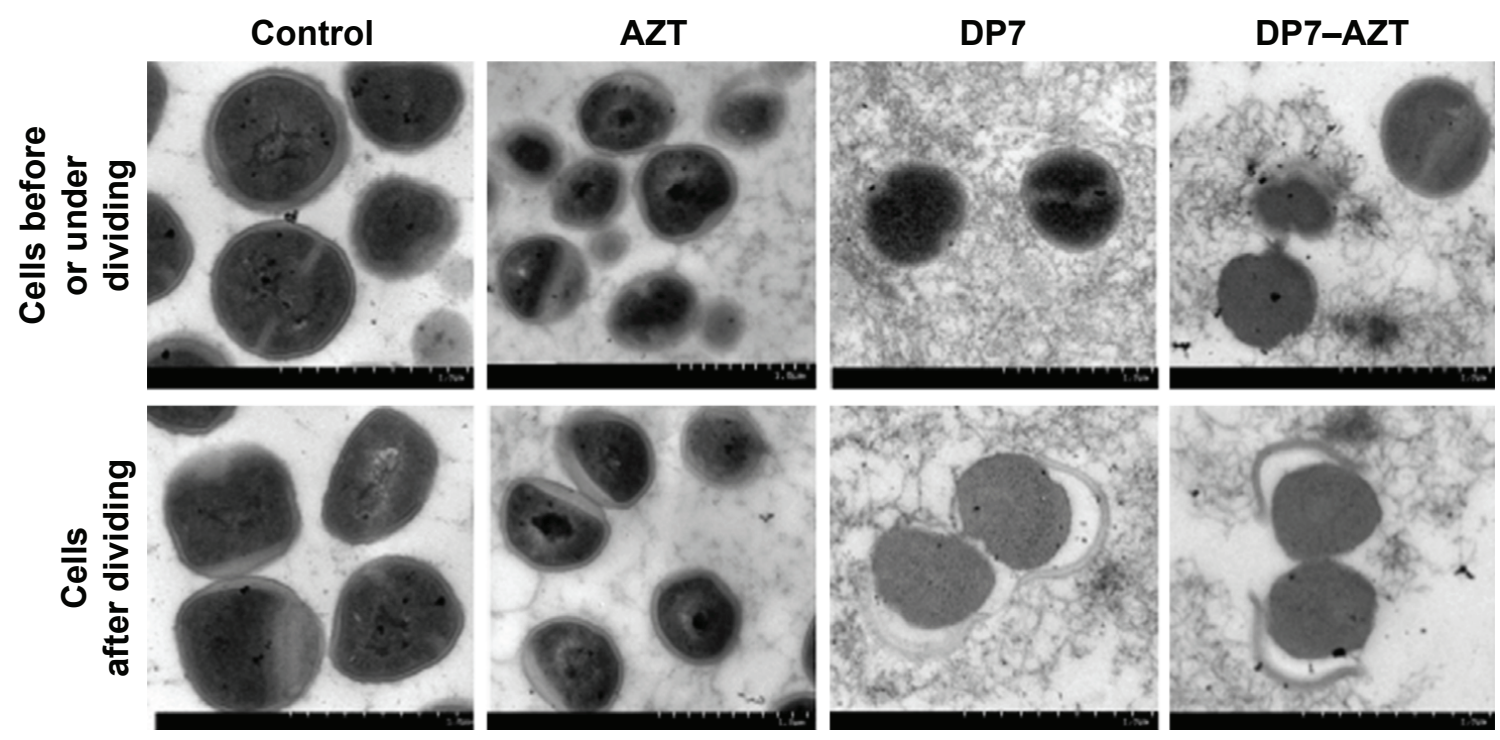

Figure I TEM image of S. aureus strain S5375.

Notes: Bacteria in all groups were round, but the AZT-treated cells had darker nuclei than the control and contracted cytoplasm. The DP7-treated cells had granular cytoplasm and fuzzy cell walls, and cell wall synthesis was interrupted in the dividing cells. DP7-AZT-treated bacteria looked most like those treated with DP7 alone, but more bacteria lacked cell walls.

Abbreviations: TEM, transmission electron microscopic; S. aureus, Staphylococcus aureus; AZT, azithromycin.

higher than those of antibiotics, ${ }^{1,4}$ AMPs are still a promising group of antimicrobial drugs, and AMP-antibiotic combinations have also effectively overcome many drug-resistant bacteria in recent years. ${ }^{5-7}$

Several studies have shown that antibacterial drugs with similar mechanisms or that influence the same target exert synergistic effects more readily than combinations of very different drugs, and several synergistic mechanisms have been analyzed. However, in this study, DP7 exerted a highly synergistic effect when combined with AZT or VAN. The antibacterial mechanism of AZT involves its combination with 50S ribosomal RNA (rRNA), thus suppressing the transcription process. However, VAN inhibits cell wall synthesis in gram-positive bacteria by binding to D-alanine residues in the glycopeptide chain. Therefore, it is quite strange that DP7 exerts highly synergistic effects with two antibiotics that have totally different mechanisms. The antimicrobial mechanism of GEN involves its binding to 30S rRNA, which is similar to that of AZT, but GEN showed almost no synergy with DP7. It is noteworthy that VAN is only functional against grampositive bacteria but acted synergistically with DP7 against several gram-negative bacteria in this study. When combined with our TEM results, these data indicate that as well as its cell wall-disrupting function, DP7 has a complex molecular-level antimicrobial mechanism, which requires investigation.

The most frequent VAN-resistance genes are the van genes, ${ }^{24,25}$ and the most frequent AZT-resistance genes are the $\mathrm{erm}$ genes. ${ }^{26,27}$ In this study, neither vanB nor $\operatorname{van} C$ was detected, while several AZT-resistance genes were detected. Genes ermA, ermB, and erm C encode methyl transferases that can change the structure of rRNA, and the altered AZT-binding site is responsible for the bacterium's resistance to AZT. Resistance genes $m s r A$ and $m e f A$ encode a macrolide efflux pump system. ${ }^{26,28,29}$ Our analysis showed that the ermB and erm $C$ genes were most common in the AZT-resistant isolates and two or more erm genes appeared together quite frequently (12/22). However, neither $m s r A$ nor $m e f A$ was related to the AZT resistance of the clinical isolates tested. This result is consistent with other studies of the relative resistance conferred by different genes. ${ }^{29}$ In this study, the synergistic activity of AZT showed that the synergistic effect of AZT-DP7 was greatest in the most highly resistant bacteria, but among the eight $S$. aureus isolates sensitive to VAN, VAN-DP7 exerted synergistic effects on three and additive effects on four. Therefore, an antibiotic-DP7 combination also exerted highly synergistic effects against antibiotic-sensitive bacteria. Although the target strain was not drug resistant, the sub-MIC concentration used in the drug combination also reduces the toxicity or adverse effects of the antibiotic and prevents the development of drug resistance. ${ }^{2}$

\section{Conclusion}

Several drug combination studies published in recent years were strain or clone specific,,$^{30,31}$ but a drug or drug combination that is therapeutic against many types of antibiotic-resistant bacteria is ideal. However, further studies 
of DP7 must be undertaken. This study not only confirmed that AMPs are broad-spectrum antimicrobial drugs effective against multidrug-resistant bacteria but also suggests that when antibiotics are combined with the AMP DP7, some antibiotic-resistant bacteria can be eradicated. However, several antibiotic-sensitive bacteria were most sensitive to the combination, and several antibiotics that only act against gram-positive bacteria acted across a broader spectrum when administered in combination with an AMP.

\section{Acknowledgments}

The study was mainly supported by the National Major Scientific and Technological Special Project for "Significant New Drugs Development" (grant no 2013ZX09102030) and the National Natural Science Foundation of China (grant no 81171780).

\section{Disclosure}

The authors report no conflicts of interest in this work.

\section{References}

1. Fuchs PC, Barry AL, Brown SD. In vitro antimicrobial activity of MSI-78, a magainin analog. Antimicrob Agents Chemother. 1998;42(5): 1213-1216.

2. Andersson DI, Hughes D. Microbiological effects of sublethal levels of antibiotics. Nat Rev Microbiol. 2014;12(7):465-478.

3. Makobongo MO, Gancz H, Carpenter BM, McDaniel DP, Merrell DS. The oligo-acyl lysyl antimicrobial peptide C(1)(2)K-2beta(1)(2) exhibits a dual mechanism of action and demonstrates strong in vivo efficacy against Helicobacter pylori. Antimicrob Agents Chemother. 2012;56(1):378-390.

4. Ge Y, MacDonald DL, Holroyd KJ, Thornsberry C, Wexler H, Zasloff M. In vitro antibacterial properties of pexiganan, an analog of magainin. Antimicrob Agents Chemother. 1999;43(4):782-788.

5. Livermore DM, Warner M, Mushtaq S. Activity of MK-7655 combined with imipenem against Enterobacteriaceae and Pseudomonas aeruginosa. J Antimicrob Chemother. 2013;68(10):2286-2290.

6. Park Y, Kim HJ, Hahm KS. Antibacterial synergism of novel antibiotic peptides with chloramphenicol. Biochem Biophys Res Commun. 2004;321(1):109-115.

7. Giacometti A, Cirioni O, Kamysz W, et al. In vitro activity of MSI-78 alone and in combination with antibiotics against bacteria responsible for bloodstream infections in neutropenic patients. Int $J$ Antimicrob Agents. 2005;26(3):235-240.

8. Cirioni O, Silvestri C, Ghiselli R, et al. Protective effects of the combination of alpha-helical antimicrobial peptides and rifampicin in three rat models of Pseudomonas aeruginosa infection. J Antimicrob Chemother. 2008;62(6):1332-1338.

9. Dosler S, Karaaslan E. Inhibition and destruction of Pseudomonas aeruginosa biofilms by antibiotics and antimicrobial peptides. Peptides. 2014;62:32-37.

10. Dosler S, Karaaslan E, Alev Gerceker A. Antibacterial and anti-biofilm activities of melittin and colistin, alone and in combination with antibiotics against Gram-negative bacteria. J Chemother. Epub 2015 Mar 24.

11. Reffuveille F, de la Fuente-Nunez C, Mansour S, Hancock RE. A broadspectrum antibiofilm peptide enhances antibiotic action against bacterial biofilms. Antimicrob Agents Chemother. 2014;58(9):5363-5371.
12. de la Fuente-Nunez C, Reffuveille F, Mansour SC, et al. D-enantiomeric peptides that eradicate wild-type and multidrug-resistant biofilms and protect against lethal Pseudomonas aeruginosa infections. Chem Biol. 2015;22(2):196-205.

13. de la Fuente-Nunez C, Cardoso MH, de Souza Candido E, Franco OL, Hancock RE. Synthetic antibiofilm peptides. Biochim Biophys Acta. 2016;1858(5):1061-1069.

14. Anantharaman A, Rizvi MS, Sahal D. Synergy with rifampin and kanamycin enhances potency, kill kinetics, and selectivity of de novodesigned antimicrobial peptides. Antimicrob Agents Chemother. 2010; 54(5):1693-1699.

15. Desbois AP, Coote PJ. Bactericidal synergy of lysostaphin in combination with antimicrobial peptides. Eur J Clin Microbiol Infect Dis. 2011;30(8):1015-1021.

16. Wu X, Wang Z, Li X, et al. In vitro and in vivo activities of antimicrobial peptides developed using an amino acid-based activity prediction method. Antimicrob Agents Chemother. 2014;58(9):5342-5349.

17. Roscia G, Falciani C, Bracci L, Pini A. The development of antimicrobial peptides as new antibacterial drugs. Curr Protein Pept Sci. 2013;14(8):641-649.

18. Hilchie AL, Wuerth K, Hancock RE. Immune modulation by multifaceted cationic host defense (antimicrobial) peptides. Nat Chem Biol. 2013;9(12):761-768.

19. JA M. Synergism testing: broth microdilution checkerboard and broth macrodilution methods. In: Garcia LS, editor. Clinical Microbiology Procedures Handbook. 2nd ed. Washington, DC: ASM Press; 2007: $1-23$.

20. Hauser C, Hirzberger L, Unemo M, Furrer H, Endimiani A. In vitro activity of fosfomycin alone and in combination with ceftriaxone or azithromycin against clinical Neisseria gonorrhoeae isolates. Antimicrob Agents Chemother. 2015;59(3):1605-1611.

21. Deslouches B, Gonzalez IA, DeAlmeida D, et al. De novo-derived cationic antimicrobial peptide activity in a murine model of Pseudomonas aeruginosa bacteraemia. J Antimicrob Chemother. 2007;60(3):669-672.

22. Kim H, Jang JH, Kim SC, Cho JH. De novo generation of short antimicrobial peptides with enhanced stability and cell specificity. $J$ Antimicrob Chemother. 2014;69(1):121-132.

23. Scott MG, Dullaghan E, Mookherjee N, et al. An anti-infective peptide that selectively modulates the innate immune response. Nat Biotechnol. 2007;25(4):465-472.

24. Gold HS. Vancomycin-resistant enterococci: mechanisms and clinical observations. Clin Infect Dis. 2001;33(2):210-219.

25. Courvalin P. Vancomycin resistance in gram-positive cocci. Clin Infect Dis. 2006;42(suppl 1):S25-S34.

26. Zmantar T, Bekir K, Elgarsadi SI, Hadad O, Bakhrouf A. Molecular investigation of antibiotic resistance genes in methicillin resistant Staphylococcus aureus isolated from nasal cavity in pediatric service. Afr J Microbiol Res. 2013;7(34):4414-4421.

27. Schmitz FJ, Sadurski R, Kray A, et al. Prevalence of macrolide-resistance genes in Staphylococcus aureus and Enterococcus faecium isolates from 24 European university hospitals. $J$ Antimicrob Chemother. 2000;45(6):891-894.

28. Yin J, Yu S, Liu X, et al. Prevalence and mechanism of resistance to antimicrobial agents in group $\mathrm{G}$ streptococcal isolates from China. Antimicrob Agents Chemother. 2011;55(1):402-404.

29. Ding ZF, Zhang H, Tang W, et al. Methylase genes-mediated erythromycin resistance in Staphylococcus aureus from bovine mastitis in China. Isr J Vet Med. 2012;67(3):170-179.

30. Furuya R, Koga $Y$, Irie $S$, et al. In vitro activities of antimicrobial combinations against clinical isolates of Neisseria gonorrhoeae. J Infect Chemother. 2013;19(6):1218-1220.

31. Barbee LA, Soge OO, Holmes KK, Golden MR. In vitro synergy testing of novel antimicrobial combination therapies against Neisseria gonorrhoeae. J Antimicrob Chemother. 2014;69(6):1572-1578. 


\section{Publish your work in this journal}

Drug Design, Development and Therapy is an international, peerreviewed open-access journal that spans the spectrum of drug design and development through to clinical applications. Clinical outcomes, patient safety, and programs for the development and effective, safe, and sustained use of medicines are a feature of the journal, which

has also been accepted for indexing on PubMed Central. The manuscript management system is completely online and includes a very quick and fair peer-review system, which is all easy to use. Visit http://www.dovepress.com/testimonials.php to read real quotes from published authors.

Submit your manuscript here: http://www.dovepress.com/drug-design-development-and-therapy-journal 